\title{
Población rural y consumo de lo rural de la región Este de Antioquia - Colombia'
}

\author{
Eryka Torrejón Cardona² y Carolina Mesa Restrepo 3
}

\begin{abstract}
RESUMEN
¿Cómo se distribuye la población rural de la región del Este de Antioquia, Colombia?, y ¿qué efectos tiene para el desarrollo local de la región esta distribución? son las preguntas de este artículo. Para responderlas se usó una metodología cualitativa basada en un estudio de caso interpretativo y comparativo, empleando técnicas como la revisión documental y la entrevista semi estructurada. Se compone de una primera parte que incluye algunos aportes teóricos del tema; en la segunda se presentan los resultados y en la tercera parte se exponen las conclusiones. Los resultados indican que hay una población rural significativa (47\%) con una desigual distribución de la propiedad y la renta rural (Gini de 0.50) y con diferentes estilos de vida y formas de consumo. En conclusión, las obras de infraestructura en conjunto con la renta rural están generando una segregación socio espacial local e intra regional en el Este de Antioquia.
\end{abstract}

Palabras clave: Población rural, región, neorurales, campesinos.

\begin{abstract}
How is the eastern Antioquia's Rural population distributed throughout the region? What effect does this distribution have for the region local development? Those are the questions for this article. A comparative and interpretative case study was used for qualitative methodology to answer the question above. Documental revision and semistructured interviews were techniques used during research. This article is composed in three parts: the first part is a basic presentation of some theories that contribute to the issues. The second part is comprised of result and third part includes conclusions. The result indicates that there is a significant rural population (47\%) with an uneven of property and rural rent (Gini of 0.50) and different lifestyle and consuming habits. This leads to the conclusion that the infrastructure works and rural rent are creating region of Antioquia zones of spatial segregation inside the Eastern region of Antioquia.
\end{abstract}

Key Words: Rural population, region, new comers, peasants.

Resultado de la investigación "Políticas de desarrollo rural, un estudio de caso comparado entre el sur de Brasil y el Oriente de Antioquia". Con recursos de la Convocatoria del Área de Ciencias Sociales, Humanidades y Artes, 2013 de la Universidad de Antioquia (Colombia) y Estrategia de Sostenibilidad 2016-2017. Artículo recibido el 15 de febrero de 2016, aceptado el 25 de mayo de 2016 y corregido el 24 de agosto de 2016. Departamento de Sociología, Universidad de Antioquia (Colombia). E-mail: etorrejon1@gmail.com

Programa de Doctorado Ciencias Sociales, Universidad de Antioquia (Colombia). E-mail: mesa_carolina@yahoo.fr 
En las últimas décadas, la población rural de la región del Este de los Andes de Antioquia-Colombia se ha distribuido acorde al desarrollo de macro proyectos -viales, industriales e hidroeléctricos -, la dotación de servicios sociales y la implementación de una mayor economía de servicios en detrimento de una economía agraria. Esta conjugación de factores generó una distribución de la poblacional rural diferenciada, no solo marcada por la producción de capital, sino por la concentración y reproducción de capital en unas subregiones más que en otras.

El artículo se compone de tres partes. En la primera se presenta el enfoque teórico y algunos aportes referidos al tema de la migración de la ciudad al campo, en la segunda se exponen los principales resultados y en la tercera las conclusiones. Responde a las preguntas: ¿cómo se distribuye la población rural de la región del Este de Antioquia? y ¿qué efectos tiene para el desarrollo local de la región esta distribución?

Se desarrolla con una metodología cualitativa basada en un estudio de caso de tipo interpretativo y comparativo, con técnicas como la revisión documental y la entrevista semiestructurada. Las entrevistas se realizaron bajo criterios de representatividad subregional, edad, sexo, oficio y tiempo de residencia en la región. Así, se realizaron entrevistas para "personas nativas" -nacidas y con residencia actual-, "residentes permanentes" -nacidos en otras regiones y con residencia en las últimas dos décadas- y "nuevos pobladores" -nacidos en otra región y con residencia en la última década-, hombres y mujeres, jóvenes -entre los 18 y 30 años- y adultos -entre los 30 y 70 años de edad.

En su parte teórica, este artículo presenta algunos de los aportes de autores que han abordado la nueva ruralidad ${ }^{4}$ como base teórica para el análisis de las poblaciones que ocupan los espacios rurales, tanto desde los espacios rurales cercanos a las grandes centralidades como de los espacios rurales más alejados y desconectados de los centros urbanos -ruralidad profunda- (Nates \& Raymond, 2007; Entrena, 1998, 2012; Fernández, 2013), acorde a los aportes que han permitido que los estudios rurales se hayan revitalizado desde la teoría clásica de las ciencias sociales (Perrier-Cornet, 2002; Pradilla, 2002; Pérez, 2004; Llambí y Pérez, 2007; Pérez et al., 2008; Entrena, 1998, 2012; Giarraca, 2001, 2004; Baigorri, 2007).

Para Ruiz y Delgado (2008), por ejemplo, la nueva ruralidad varía según la disciplina, los ensayos que la desarrollan son analíticos, descriptivos o normativos. Ávila (2015) rescata la perspectiva de la geografía rural como base de la nueva ruralidad, y para Pradilla (2002) la nueva ruralidad es aún polisémica y confusa. Estos debates, aún sin clausurar, permiten indicar que no hay un consenso general y al unísono sobre el enfoque, teorías o métodos que caractericen a la nueva ruralidad.

La reelaboración teórica de los estudios rurales determinará en este sentido las categorías de análisis que definen los perfiles de nuevos y antiguos pobladores de zonas rurales. Los estudios

Según Llambi y Pérez (2007), la nueva ruralidad tiene como base la teoría de las transiciones, la teoría de la agencia de los actores sociales y la teoría espacial. La teoría de las transiciones alude al análisis histórico de los procesos, el tiempo y el espacio son categorías básicas para comprender los cambios de acuerdo a un contexto y momento determinado. La teoría de la agencia de los actores sociales privilegia la capacidad de acción colectiva y de praxis social individual o de grupos sobre las condiciones macro estructurales y la teoría del espacio permite. desde el enfoque territorial, estudiar las relaciones escalares entre lo local y lo global. Este enfoque permite, a la vez, el análisis integrado de lo rural y lo urbano. 
llevados a cabo sobre las nuevas ruralidades buscarán sobrepasar la dicotomía campo/ciudad, conceptualizando así los espacios rurales como un continuum urbano dentro de una sociedad global (Kayser, 1996) o, incluso, como el "jardín de la urbe global" (Baigorri, 2007). La globalización económica, tecnológica y cultural marca una tendencia de homogeneización de las sociedades, y tanto desde sectores económicos en plena expansión -por ejemplo el ecoturismo o el turismo rural-, como desde el rechazo evidente de algunos urbanitas por la vida de la ciudad, el consumo de la naturaleza y de los espacios rurales es cada vez más intenso.

Para autores como Baigorri (2007) y Kayser (1996), el espacio rural no puede definirse únicamente por una vocación exclusivamente agrícola o extractivista, sino también por toda una serie de usos del suelo que difieren de las actividades de producción. El incremento de las amenidades que ofrecen actualmente estos espacios para la instalación de nuevos habitantes urbanitas, lleva a estos autores a elaborar un análisis centrado en los flujos económicos, culturales y tecnológicos que circulan: si bien no existen límites de orden geográfico, estos son de orden social.

Para Kayser (1996), los espacios rurales en Francia, sean estos periféricos, centrales o periurbanos, están integrados al sistema socio-espacial global, del cual los primeros son un componente más. De este modo, la expansión de las infraestructuras, la disposición en servicios básicos y en telecomunicaciones, facilita la instalación de los neorurales 5 . Por esta razón, en su análisis sobre la configuración y el nacimiento de los nuevos campos franceses, aparece como preponderante la cuestión de la distribución espacial de las amenidades: para el urbanita, por lo general de clase media y media alta, el hecho de instalarse en el campo no se da de manera espontánea, se trata de un proyecto planeado y este espacio debe poder permitir al neorural reproducir varias de las condiciones de su anterior estilo de vida.

En España, Baigorri nos describe la urbe global como el resultado de un "proceso de urbanización general del espacio", a través del crecimiento demográfico y espacial de las ciudades y del traslado de la cultura urbana hacia los espacios rurales. Sin embargo, operar una distinción entre espacio rural y espacio urbano es arbitrario para Baigorri, pues las diferencias en hábitos, actitudes o valores serían cada vez menos tajantes, incluso en lo que a modos de producción se refiere. Los intersticios de la urbe global serían lo que este autor denomina la Ruralia, y el papel de esta ya no consiste exclusivamente en alimentar las ciudades, sino que cumple "funciones mucho más complejas: entre las cuales alimentar el espíritu es una de las más importantes; casi la misma función que, en términos de micro-urbanismo, cumplieron los parques y las zonas verdes en la ciudad industrial" (Baigorri, 2007: 18). Por otro lado, el rural viene siendo progresivamente asumido como un "actor multisectorial".

En balance, los aportes desde la nueva ruralidad para analizar la migración por amenidades -servicios rurales- y la movilidad residencial desde las zonas urbanas hacia las zonas rurales, permiten contar con categorías analíticas y marcos metodológicos que posibilitan describir y analizar los cambios que acontecen en las poblaciones de las zonas rurales bajo el desarrollo

Una presentación, clasificación y caracterización del neorural se encuentra en detalle en Biagorri (2007) y Morales Prieto (2016). Para este artículo, los neorurales son asumidos como personas que han hecho cambios de residencia de una zona urbana a una zona rural motivados por razones ambientales, económicas, políticas y/o culturales, regularmente los cambios de residencia y sus motivaciones no cuentan con un registro en bases de datos agregadas -tipo empadronamiento- para el caso de Colombia. 
capitalista global, sean estos cambios desde unos nuevos campesinos y/o desde los pobladores neorurales.

Así, en Argentina (Giarraca, 2001, 2004; Barros, 2006; Clementi, 2012; Craviotti, 2014; Merlotto et al., 2012) abordan los cambios en los usos del suelo rural y las transformaciones de la economía agrícola. Las autoras, desde diferentes rutas disciplinarias, describen los cambios en la renta del suelo y los efectos en la dinámica territorial en la relación urbana y rural, así como la densificación y gentrificación de viviendas en los complejos residenciales ubicados en las zonas rururbanas de Buenos Aires. En este sentido, Barros (2006), por ejemplo, indica que la identidad del lugar de los pobladores de estas zonas no siempre es armónica y comunitaria. Reflexiones similares se encuentran en los aportes sobre exclusión y gentrificación rural que hace Clementi (2012) y Craviotti (2014). Giarraca $(2001,2004)$, por otra parte, presenta los procesos de migraciones estacionales y residenciales por la actividad cañera azucarera en la región de Tucumán.

Los aportes desde México (Pradilla, 2002; Ramírez, 2003; Cruz, 2009 y Bonilla, 2008) se centran en presentar las desigualdades sociales imperantes tanto en Ciudad de México como en otros Estados. Ramírez (2003) y Cruz (2009), desde la sociología rural, muestran la influencia de los procesos de industrialización y urbanización en las poblaciones rurales cercanas a la Ciudad de México; mientras que Bonilla (2008) nos presenta, bajo el caso del cultivo del nopal, la persistencia de la pobreza rural en zonas con bajos niveles de urbanización. Pero es quizás Pradilla (2002) quien nos presenta mejor el contexto de la urbanización acelerada de México en la década de 1990, la permanente y decisiva influencia de las relaciones campo y ciudad y, con esto, las formas de pobreza y exclusión generalizadas que se expresan no solo en el campo, sino ahora extendidas en más zonas de la ciudad.

En Colombia, desde los casos de Manizales, Bogotá, La Calera, Cajicá, Eje Cafetero y Medellín, se muestran algunas características de las poblaciones rurales (Nates, 2008; Méndez, 2012, 2013; González, 2009; Castañeda, 2012; Ortíz Hernández, 2015; Fernández et al., 2013; Arango, 2008; Cadavid, 2009; García \& Gálvez, 2011; Agudelo, 2012; Castaño, 2014).

Méndez (2012) hace una tipología de los nuevos pobladores rurales, y Nates (2008) indica los intereses personales y colectivos por migrar de la ciudad al campo y los intereses mágico-religiosos que los motivan, para el caso de Manizales. Otros estudios, entre estos el de González (2009), retoman los proyectos residenciales y la relación con el recurso hídrico para explicar la fragilidad y alteración de algunos ecosistemas del eje cafetero a causa de la expansión de viviendas en forma de condominios o casas aisladas. Y en La Calera, Bogotá, Castañeda (2012) explora las dinámicas de las familias rurales y campesinas.

Ortíz Hernández (2015) y Fernández et al. (2013) explican el proceso de la migración de la ciudad al campo a partir de discursos y prácticas sociales asociadas a un nuevo indigenismo. Retoman el caso de Bogotá, Cajicá y otros municipios de Cundinamarca a partir de la instalación de los complejos residenciales con baja densidad, pero con una alta renta urbana y la gentrificación rural por efectos de la conurbanización de Bogotá y de ciudades intermedias.

Arango (2008), Cadavid (2009), García \& Gálvez (2011), Agudelo (2012) y Castaño (2014), para el caso de Medellín, presentan tanto las visiones nostálgicas de la vida campesina frente a otras visiones en los procesos de metropolización regional y, a la vez, indican la necesidad de regular 
los usos del suelo de las zonas rurales frente a la expansión urbana de la ciudad. Estos aportes, en general, están subrayando el uso de elementos emblemáticos de una cultura campesina andina como valores locales y en contracorriente con el crecimiento económico y de infraestructura que ha tenido la ciudad en las últimas dos décadas.

Coinciden los autores de Argentina, México y Colombia en explorar las características de las poblaciones rurales, en la actualidad, en las ciudades metropolitanas como Buenos Aires, México D.F y Bogotá, y de regiones con una economía agrícola en permanente transición, para los casos de Tucumán en Argentina, Milpa Alta en México y Cundinamarca y Eje Cafetero en Colombia, respectivamente.

En resumen, los aportes, en especial de Kayser (1996), Pérez (2004), Pérez et al. (2008), Nates \& Raymond (2007), Nates (2008) y Baigorri (2007) sobre los nuevos campesinos, la revitalización del campo, la búsqueda de la naturaleza y los nuevos campos, son procesos que se vienen generalizando en varios países. Los autores coinciden en presentar, desde diferentes disciplinas, los cambios en las poblaciones rurales con manifestaciones como la migración por amenidades, la movilidad residencial de la ciudad al campo, la segregación socio espacial y una recomposición poblacional a partir de la transición de una economía agrícola hacia una economía multisectorial.

Los aportes indican así que características como la actividad agraria, los tipos de familia y los perfiles ocupacionales de las poblaciones rurales, han cambiado como resultado de los procesos de metropolización y de post industrialización. Las razones de la migración por amenidades y de la movilidad residencial hacia las zonas rurales son, desde intereses ambientales, ecológicos, de bienestar y salud, hasta intereses políticos, ideológicos y mágico-religiosos, como manifestación de cierto desencantamiento con el modelo de desarrollo económico de las ciudades.

Las poblaciones rurales en las últimas décadas vienen en una permanente reconstrucción, la cual es necesario explorar y analizar desde diferentes contextos y en consonancia con los estudios citados de Chile (González et al., 2009; Salazar et al., 2013), Argentina, Colombia, México, Estados Unidos (Hines, 2010, 2012) y Canadá (Guimond \& Simard, 1998, 2010).

\section{Área de estudio}

El Este es la región más representativa en el desarrollo de obras de infraestructura vial en Antioquia -la autopista Medellín-Bogotá, el Aeropuerto Internacional y la Zona Franca. Es considerada geoestratégica para el desarrollo del departamento y del país en los renglones de infraestructura, producción de energía, producción agropecuaria, industrial y minera. Esto la ha situado a la vanguardia en materia de infraestructura vial a nivel nacional y la posiciona como una de las economías regionales ${ }^{6}$ con mayor diversidad de actividades comerciales y en la exportación de productos como flores, textiles y café (Instituto de Estudios Regionales, 2000, García \& Aramburo, 2011). Político-administrativamente el Este se divide en cuatro subregiones, compuestas por veintitrés (23) municipios: Altiplano (9), Bosques (3), Embalses (7) y Páramos (4) (Figura № 1).

Una exposición detallada del surgimiento y la expansión de la industrialización de Antioquia a principios del siglo XX y el papel de la geografía y la cultura de la montaña -ethos paisa- se encuentra en la obra de Parsons (2005). 
Figura $\mathrm{N}^{\circ} 1$

Mapa de ubicación de Antioquia en Colombia

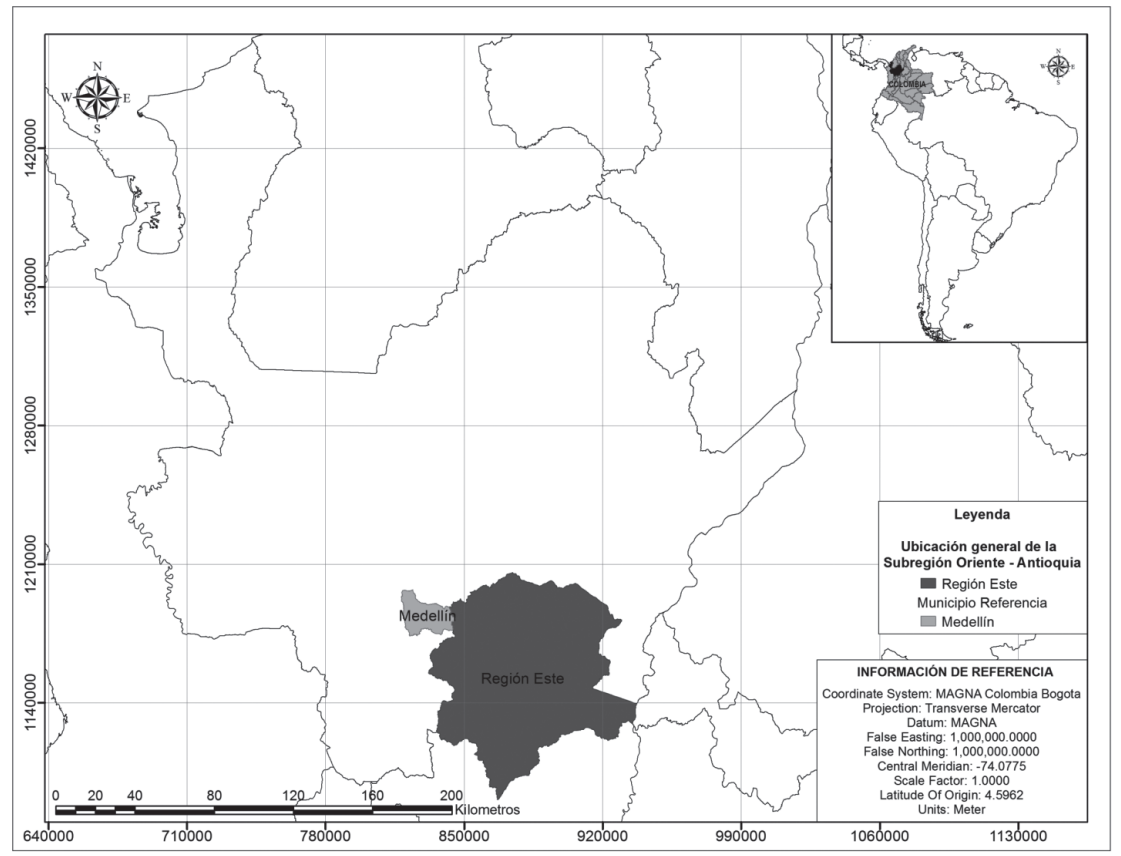

Fuente: Elaboración propia.

El grueso de la actividad comercial se concentra en los municipios del Altiplano, de ahí que la actividad agrícola en las demás subregiones se caracterice por la falta de mercado para sus productos y la baja renta. En consecuencia, el desarrollo económico de la región y sus beneficios no es igual para todas las localidades, dado que, en términos de incremento del ingreso y acceso a oportunidades, existen marcadas diferencias entre municipios y entre subregiones. Esto se expresa por ejemplo en el Gini Rural (Figura N²).

La región tiene una extensión de 7.021 kilómetros cuadrados. La población es de 540.184 personas, distribuidas en 286.813 (53\%) en la zona urbana y 253.371 (47\%) en la zona rural. El Censo de Población y Vivienda del año 2005 muestra que cerca del $40 \%$ de sus habitantes vive en condiciones de pobreza, y un 20\% en situación de miseria (DANE, 2005, Gobernación de Antioquia, 2009).

Por subregiones, de mayor a menor población, se encuentra que el Altiplano tiene una población total de 353.414 personas, 216.019 son de la zona urbana y 137.395 de la zona rural. La subregión de Páramos cuenta con una población de 84.698 personas, 27.220 personas en la zona urbana y 57.478 en la zona rural. La subregión de Embalses tiene una población de 69.549 personas, 32.598 en la zona urbana y 36.951 en la zona rural, y la subregión de Bosques cuenta con una población de $32.523,10.976$ personas en la zona rural y 21.547 en la zona rural (DANE, 2005) (Figura $\mathrm{N}^{\circ} 3$ ).

Los datos demográficos disponibles del Censo de Población y Vivienda del 2005 no permiten identificar directamente los cambios de residencia del Este de Antioquia desde las zonas urbanas 
Figura $N^{\circ} 2$

Mapa de Gini Rural

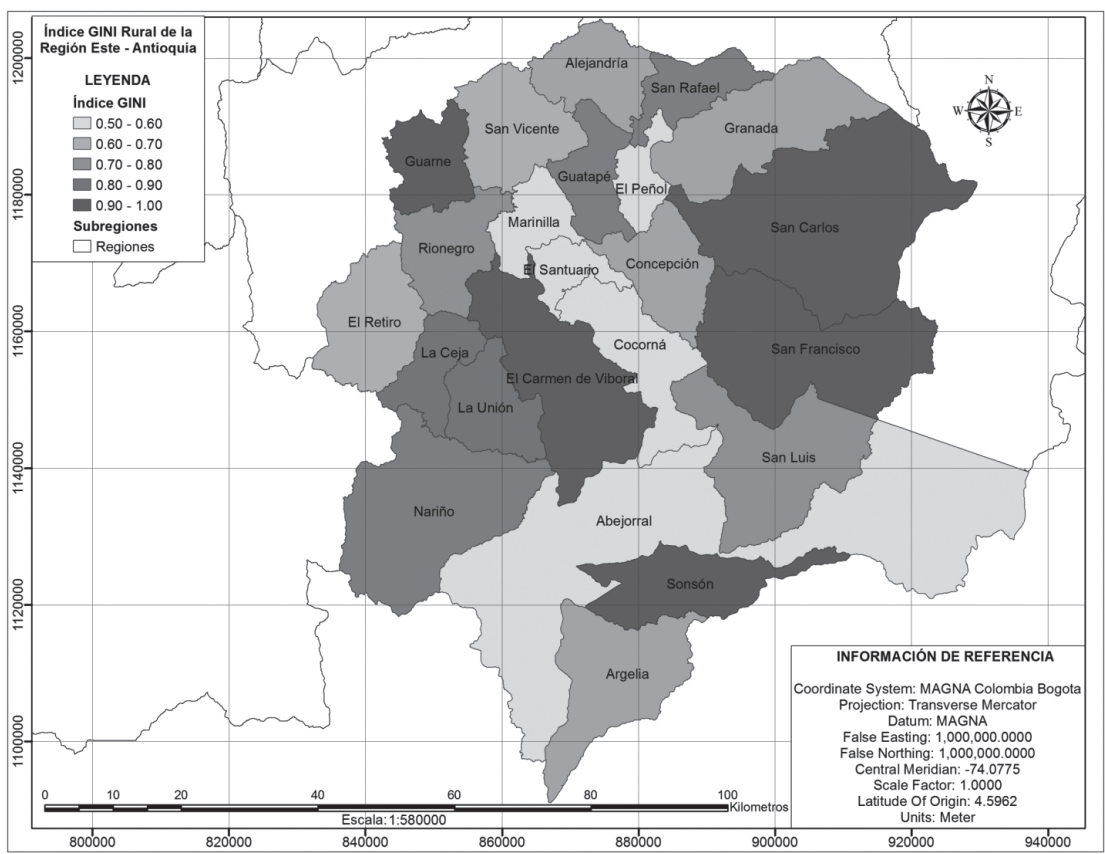

Fuente: Elaboración propia.

Figura $\mathrm{N}^{\circ} 3$

Mapa de Población

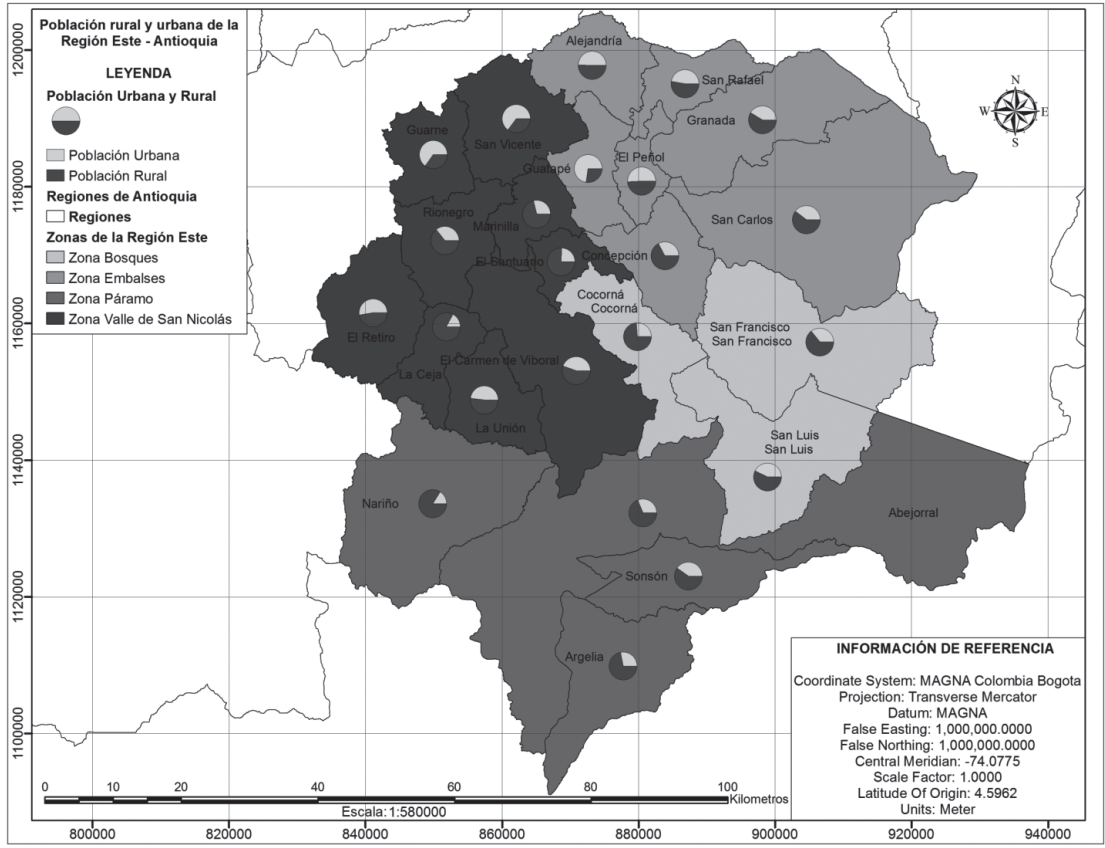

Fuente: Elaboración propia. 
hacia las zonas rurales, ni por localidad o intraregional en forma longitudinal y comparativa en el tiempo, debido a que no existe una variable que la represente en los censos anteriores.

Sin embargo, datos indirectos como los referidos a la variable ¿lugar donde vivía hace cinco años?, incluida en el Censo de Población y Vivienda del 2005, pueden aportar algunos datos representativos: 26.833 personas de las zonas rurales del Altiplano indican que sí habían realizado cambios en los últimos cinco años; así como 12.408, 8.671 y 6.569 personas de las zonas rurales para las subregiones de Páramos, Embalses y Bosques, respectivamente (DANE, 2005).

Por la subregión de Bosques, sobresalen los datos de las personas de las zonas rurales de San Carlos (2.789) y Granada (2.232) que expresaron haber hecho cambios de lugar en los últimos cinco años. Y las localidades emblemáticas del Altiplano, Rio Negro y Guarne, con 6.769 y 6.106 personas, respectivamente.

Los datos demográficos disponibles para la región del Este de Antioquia, por fuentes como el Censo Nacional de Población y Vivienda, presentan la distribución poblacional por zona rural y urbana para el 2005, pero son poco útiles para describir y analizar la dinámica poblacional y, en forma concreta, el movimiento migratorio de la ciudad al campo o del campo a la ciudad, como sí es posible hacerse en otros contextos.

Uno de los sucesos poblacionales que marcó la región Este de Antioquia en las últimas décadas es la migración forzada -desplazamiento forzado- por causa del conflicto armado. El registro de tierras abandonadas o despojadas, disponibles en La Red Nacional de Información (2015) y su Registro Único de Victimas (RUV), indican que el Este de Antioquia, entre los años 1995 al 2005, produjo el mayor número de población desplazada (103.467), población que representó el 35\% de víctimas del conflicto armado en todo el departamento de Antioquia.

El conflicto armado en la región se agudizó entre los años 1995 y el 2005, y en consecuencia se produjo el desplazamiento forzado con el eventual abandono y despojo de tierras (Fajardo, 2000; Uribe, 2001; García \& Aramburo, 2011). Las subregiones con mayor número de desplazados forzados fueron Bosques y Páramos, y son estas mismas subregiones donde se registran las mayores áreas de tierras abandonadas y despojadas. Así, las fuentes muestran cómo los índices de desplazamiento forzado empiezan a disminuir en la totalidad de los municipios a partir del 2005.

Sin embargo, todos estos datos son ilustrativos, ya que no permiten describir y comparar ${ }^{7}$ en forma regional, subregional y temporal -1995, 2005 y 2015- el ¿cómo y a dónde llegó está pobla-

\footnotetext{
En la literatura especializada, la región del Este de Antioquia, Colombia, no cuenta con investigaciones que aborden la distribución de la población rural en la historia reciente (2005-2015), tampoco en forma comparativa al interior de cada municipio o en el conjunto de las localidades de la región. En su lugar, se encuentran estudios que abordan la distribución poblacional en las escalas nacionales, supra regionales y metropolitanas.

Los estudios desde la demografía se encuentran vinculados a la migración interna y externa de la población, se suman los aportes que se dedican a describir y analizar la situación y las tendencias de la movilidad (espacial) interna a nivel de datos poblacionales nacionales, departamentales y metropolitanos (Martínez, 2002), y a la movilidad poblacional forzada -desplazamiento forzado o refugiados internos- (Uribe, 2001; Fajardo, 2000; Ruíz, 2008).
} 
ción desplazada?, debido al inevitable subregistro que presentan las fuentes en contextos donde aún el conflicto armado es permanente ${ }^{8}$.

Ahora bien, el desplazamiento forzado tiene una estrecha relación con el conflicto armado en la región y afectó principalmente a la población de las zonas rurales (47\%) caracterizadas por una economía de minifundio y con niveles de pobreza y de miseria significativos. La combinación de ambos procesos, el conflicto armado y el desplazamiento forzado, jugó como elemento determinante para el despoblamiento de unas subregiones, maximizando los efectos de la informalidad en la tenencia de la tierra de los campesinos por la ausencia de una reforma agraria efectiva y distributiva (Fajardo, 2000).

La dotación de infraestructura vial, servicios sociales y distribución de la propiedad rural es una ventaja para unas subregiones y coloca a otras en franca desventaja. Estas desigualdades aumentaron aún más a nivel subregional con la alta informalidad en la tenencia de la tierra por parte de campesinos minifundistas, el desplazamiento forzado a causa del conflicto armado y las eventuales posibilidades de un retorno y un repoblamiento de las zonas rurales más afectas durante las últimas dos décadas.

Durante casi una década (1995 a 2005) algunas zonas rurales de la subregión de Bosques y Páramos se despoblaron a causa del desplazamiento forzado y paulatinamente algunas de las familias desplazadas han retornado a sus predios, sea por iniciativa propia o por el apoyo de los programas del Gobierno'.

\section{Nativos, nuevos residentes y nuevos pobladores}

En el contexto actual y regional del Este de Antioquia, la población de las zonas rurales presenta procesos simultáneos y complejos, difíciles de evidenciar con los datos demográficos disponibles.

El primero es el abandono-despojo y retorno de la población desplazada, y el segundo, la llegada de nuevos pobladores, ambos procesos se localizan y son motivados en forma distinta. Por esta razón se asumió realizar entrevistas semi estructuradas bajo criterios como representatividad subregional, edad, sexo, oficio, lugar de nacimiento y tiempo de residencia, definiendo tres perfiles: nativos residentes -o campesinos raizales-, residentes permanentes y nuevos pobladores.

Los nativos residentes o campesinos raizales son personas que tienen vínculos ancestrales con la región desde hace una o dos generaciones -padres e hijos- y en sus testimonios se evidencia la experiencia socio espacial que han tenido en la región durante más de dos décadas:

El Gobierno de Colombia y las Fuerzas Armadas Revolucionarias del Pueblo FARC-EP, desarrollan el acuerdo de paz; sin embargo, los organismos internacionales y nacionales de derechos humanos siguen reportando hechos victimizantes, situación que afecta el registro de datos y la eventual atención integral de víctimas en condiciones de conflicto armado interno.

En especial, luego de la Ley 1448 del 2011 -Ley de Víctimas y de Restitución de Tierras (Congreso de la República Colombia)- vinculada con el reconocimiento de los derechos de las víctimas del conflicto armado, entre estos el derecho a la reparación de sus tierras. 
"...en la zona de arriba, el altiplano, que llevo veinte y un años he notado pues la transformación... Estamos muy cerquita de la cultura citadina... Uno no nota quién es campesino y quién es de acá. Los jóvenes sobre todo ya se parecen.......si vamos al campo propiamente, vemos que hay muy pocos jóvenes... La población adulta también se ha ido transformando, su manera de pensar, su manera de relacionarse con la tierra... ...es una cultura campesina en transformación y eso es natural" (Funcionaria, subregión del Altiplano).

"Los hijos de los campesinos ya no quieren ser campesinos, ya quieren es trabajar en una empresa. Ahorita está el boom de la construcción, o se van al casco urbano, entonces el campo ya no es viable para ellos" (Joven tecnólogo agropecuario, subregión del Altiplano).

"...la cultura, como son las tradiciones que teníamos los viejos de sentarnos alrededor del fogón, eso se acabó... Lo que hace que llegó la electricidad y la televisión al campo, ya no nos sentamos en familia... ¿Qué ha pasado con la apertura de las carreteras? ¿Es desarroIlo?, icierto que sí!, pero han venido un montón de gente de las ciudades y se nos han asentado en lo rural..." (Agricultor y miembro de una asociación local, subregión del Altiplano).

"Eran muy recatados (los campesinos), con costumbres muy católicas, los hijos eran obedientes, trabajadores... Ahora ya no es así...eso fue algo que a mi modo de ver se transformó después de las guerras, además ya con todo esto de la autopista y demás, ya las costumbres han cambiado... ...ya la sociedad ha cambiado" (Docente y miembro de una asociación local, subregión de Bosques).

"Trabajo en el manejo y la trasformación de la guadua como alternativa sostenible para un desarrollo poblacional... Es una vereda donde se conservan muchas tradiciones del paisa campesino tradicional... Desde que llegué aquí he sido bien recibido, como una persona, pues que le puede aportar y puede aprender de la comunidad" (Joven, gestor nacional de la Red Colombiana de Jóvenes Rurales, subregión de Bosques).

"Sí, sí ha pasado, o sea que venga gente diferente, pero igual en esta vereda todo el mundo ha acogido a los que vienen de otras partes, o sea es algo que resalta mucho de la vereda..." (Joven bachiller, agricultor, miembro de una asociación local, subregión de Altiplano).

“En cuanto a la población es lo que más ha cambiado... está más poblado, viene más gente, hay más casas, menos cultivos por todo esto de las construcciones... Se hacen menos eventos, la gente se está aislando más, ya no se reúnen bastante para hacer cosas en bien de la comunidad" (Joven bachiller, agricultora, miembro de una asociación local, subregión del Altiplano).

"Muchas de las finquitas las vendieron pensando que con la plata podían vivir y quedaron de mayordomos... Se conserva una cantidad (de campesinos) en algunas de estas poblaciones, pero ya tienen la casita en el campo con luz y con otros servicios telefónicos, que no los tenían... la vida campesina en esta zona de aquí ya es muy parecida a la de ciudad... El metro cuadrado vale casi lo mismo que en la parte urbana..." (Sacerdote, Rector de una Corporación Educativa con enfoque agroecológico, subregión del Altiplano). 
"...ya tengo internet, ya tengo televisión, tengo gas domiciliario, tengo acueducto, por mi casa pasa transporte público, dos, tres veces al día, entonces tengo la tranquilidad del campo, pero las comodidades casi de lo urbano mezclados ahí..." (Agricultor, asociación de producción agroecológica, subregión del Altiplano).

En los anteriores testimonios se evidencian cambios en los estilos de vida de padres a hijos, en los tipos y valores familiares y en los modos de producción (de una economía agraria hacia una economía de servicios). Las personas entrevistadas vinculan estos cambios a la dotación de infraestructura vial y al incremento de amenidades en la región, en especial en la subregión del Altiplano y de Embalses.

Los testimonios mantienen una estrecha relación con algunos de los procesos que han analizado autores como Kayser (1996), Pérez (2004) y Baigorri (2007) en otros contextos, y que para el caso del Este de Antioquia se expresan como un perfil de nuevos campesinos (Pérez, 2004), caracterizado por unas relaciones espacio temporales cada vez más estrechas con la sociedad global (Kayser, 1996) y donde los espacios rurales -zonas rurales- son considerados como jardín de la urbe global (Baigorri, 2007).

Ahora bien, los testimonios de las personas con un perfil de residentes permanentes - con menos de dos décadas de residencia en la región- expresan su experiencia espacio temporal con una alta valoración de servicios ambientales -capitalización de la naturaleza-, por el acceso a las amenidades y el contacto con la cultura campesina:

"Aquí tenemos la posibilidad de estudiar, de estar más activos, lo que no teníamos en Medellín, no hay que coger transporte, todo lo hacemos a pié, en Medellín hay que coger transporte para todo" (Confeccionista jubilada, subregión de Embalses).

"...en Medellín todo es caro, para todo hay que pagar, el aire está muy contaminado, mucho calor, la ciudad es un caos, hay que consumir todo el tiempo" (Joven tecnólogo agropecuario, subregión del Altiplano). “...no había dónde esconderse en Medellín, no había dónde dormir en silencio... Eso a mí me mortificaba, me mantenía con los nervios de punta, y entonces dije me voy para el campo" (Ingeniero ambiental jubilado, Subregión de Embalses).

"Cuando adquirimos el terreno ya estábamos muy metidos en todo esto de la conservación... Por cosas de la vida esa casa se creció...dijimos, esta casa quedó muy grande, algún día va a ser un gran centro pedagógico, y así fue, esa información fue mandada al cosmos" (Docente jubilada, subregión de Altiplano).

"Vamos los dos en representación de la mujer rural del Peñol... El objetivo es ayudarles, hay muchas madres adolescentes, en muchos municipios no tienen proyectos para ayudarles a ellas, muchas no saben lo que es la primaria" (Confeccionista jubilada, subregión de Embalses).

"A veces vienen y se quejan...que me va a tocar vender la tierra porque no tengo para comprar insumos...nosotros les decimos que hay otras vías y les ayudamos, si vemos que se les presentan dificultades para el comercio nosotros le compramos los productos a un precio 
justo y lo comercializamos, porque a ellos se les dificulta toda esta parte pues de internet y esas cosas actuales... queremos mostrarle a la gente que hay otros caminos productivos que antes se trabajaban, pero que se han perdido, entonces es tratar de recuperar esas raíces donde ellos estaban antes" (Joven tecnólogo agropecuario, subregión de Bosques).

De acuerdo con Lara (citado por Fernández, 2013), estos movimientos de residencia hacia las zonas rurales generan entonces "tanto una urbanización de la vida campesina, como una ruralización de la periferia urbana" (Lara, 1996, citado por Fernández, 2013: 9). No obstante, la presencia de residentes permanentes en la región del Este de Antioquia - en especial en el Altiplano y en Embalses- puede estar contribuyendo al desarrollo de nuevas formas de producción local, mejorando las condiciones de vida por medio de una agricultura amistosa con el entorno, estableciendo redes de distribución de productos orgánicos, o generando empleos en la producción agroecológica, el ecoturismo o el turismo rural.

Los nuevos pobladores son personas con menos de una década de residencia en la región, además de la alta valoración de los servicios ambientales y el acceso a las amenidades expresan motivaciones asociadas a la búsqueda de una mejor calidad de vida por medio del contacto con la naturaleza. Realizan prácticas mágico-religiosas y se caracterizan por una ideología y una estética asociadas con movimientos contraculturales o alternativos, estos a su vez identificados por planteamientos ecológicos, utópicos o igualitarios, y que buscan generar un impacto ambiental y social positivo a nivel local. Estos movimientos, en auge desde la segunda mitad del siglo XX, generan la movilidad residencial de numerosas personas de la ciudad hacia el campo (Fernández, 2013).

"Mi proyecto de vida gira en torno de un lote en donde quiero construir con una arquitectura que, me parece a mí, sustentable, sostenible, según las tendencias nuevas, las nuevas olas, en las tendencias que vienen sucediendo: cultivar su propia comida, cultivar, cosechar para comer" (Licenciado en Educación y artista, subregión de Embalses).

"Nosotros hacemos parte de un grupo de meditación, tenemos un maestro y hacemos encuentros espirituales a final de año" (Docente jubilada, subregión del Altiplano). "Acá hay muchos extranjeros que tienen finca, muchos van y se encierran, es un fenómeno que si usted analiza puede ser negativo, entonces para no crear xenofobias, es un problema que hay que pensar y analizar" (Licenciado en Educación y artista, subregión de Embalses).

De acuerdo con Nates (2009: 283), la producción del territorio implica la materialización de las "diferentes dimensiones de la cultura, tales como: la economía, la política, la religión y hasta la lúdica", al mismo tiempo que moviliza las concepciones ontológicas que se tengan del mundo y del espacio. El territorio es aprehendido así desde una cosmogonía que sitúa e identifica a los seres naturales desde unos discursos y unas prácticas determinadas.

Si se analizan los testimonios de los residentes permanentes y de los nuevos residentes desde las perspectivas de Fernández (2013) y Nates (2009), es posible darse cuenta cómo estas personas llevan consigo todo un acumulado político y cultural que les permite apropiarse y transformar el espacio rural donde han decidido vivir.

En el caso del Este de Antioquia, se encontró que la experiencia espacio temporal de los nuevos residentes se materializa en la vida cotidiana local con prácticas como la producción agro- 
ecológica y acciones de conservación ambiental, logrando incentivar y apoyar la transformación de modos de producción agraria, renovando o protegiendo los ecosistemas ${ }^{10}$, generando empleo estacionario y facilitando la integración y la capacitación de otros nuevos residentes.

\section{Conclusiones}

Los resultados, según los datos demográficos disponibles, indican que la población rural del Este de Antioquia equivale al 47\% (253.371 personas) del total poblacional (540.184 personas), es decir, aún la región tiene casi la mitad de su población ubicada en zonas rurales.

Aunque no es posible comparar y presentar datos directos que representen la migración por amenidades o cambios de residencia desde las zonas urbanas hacia las zonas rurales, los datos indirectos indican que la población rural en la región realizó cambios de residencia en el período 2000 a 2005, sobresaliendo las subregiones del Altiplano (26.833) y de Páramos (12.408), sobre un total de 54.481 personas que indicaron haber realizado cambios de residencia durante el quinquenio (2000 a 2005).

La población del Este de Antioquia se distribuye casi en forma proporcional entre las zonas rurales y las zonas urbanas, las subregiones donde hay mayor población rural son: Altiplano, Páramos, Embalses y Bosques, de mayor a menor.

Ahora, el por qué se distribuye la población rural de esta forma parece tener coincidencias con los procesos que se han mostrado en otros contextos autores como Kayser (1996), Pérez (2004), Baigorri (2007) y Nates $(2008,2009)$. Con los datos de las entrevistas se exponen las motivaciones e intereses por las cuales las personas deciden residir en las zonas rurales: los nativos residentes o campesinos nativos tienen vínculos ancestrales con el territorio y un alto conocimiento local de los procesos ecológicos y sociales que han capitalizado para permanecer durante varias generaciones. Las nuevas generaciones se caracterizan por el acceso a servicios sociales como la educación y el empleo estacionario. Se localizan tanto en zonas cercanas a los centros urbanos como en zonas alejadas.

Los residentes permanentes son personas que han decido radicarse en las zonas rurales hace dos décadas a partir de la valoración de los servicios ambientales y el intercambio cultural con las culturas campesinas. Se caracterizan por residir en forma permanente en las zonas rurales y son personas profesionales, jubiladas o con destrezas en artes y oficios. Residen en zonas cercanas a las zonas urbanas y con acceso a vías y telecomunicaciones.

Los nuevos pobladores residen en las zonas rurales hace menos de una década. Tienen motivos ecológicos, políticos, ideológicos y se caracterizan por ubicarse en zonas con acceso a amenidades como las telecomunicaciones, los servicios sociales y la infraestructura, y valoran

\footnotetext{
Se encontró el caso de una joven pareja que comercializa sus productos en la ciudad de Medellín a través de pedidos por Internet, lo cual presenta grandes similitudes con los modelos ASC (Agricultura Sostenida por la Comunidad) que se vienen desarrollando en Francia y España. En este último, este modelo es conocido con el acrónimo BAH (Bajo el Asfalto está la Huerta), y propone un nuevo sistema de producción, distribución y consumo basado en las premisas de la agroecología.
} 
en gran medida también los bienes y servicios comerciales. La territorialización de los nuevos pobladores se asocia a la pluriactividad del campo, la expansión de la urbanización en las zonas rurales y al desencanto del modelo de desarrollo, según los testimonios y en consonancia con la literatura especializada.

Según los testimonios recolectados, se encontró que los intereses y las motivaciones de los nuevos pobladores del Este de Antioquia van desde un deseo de intervención local para lograr un impacto global en el medio ambiente, la búsqueda de una mejor calidad de vida en contacto con la naturaleza, hasta la evasión de la violencia urbana. De este modo, el "desencanto de la vida citadina" se interpreta como una huida hacia un espacio "más natural o más tranquilo". Un espacio que facilite y permita realizar un cambio en el estilo de vida.

Las amenidades rurales facilitan entonces la migración de la población citadina, y en la perspectiva de la nueva ruralidad, las fronteras entre espacio urbano y espacio rural se difuminan por la expansión de dichas amenidades: el Este de Antioquia presenta de este modo una población rural que integra las diferentes expresiones de la modernidad en términos del acceso a nuevas tecnologías y a ofertas culturales, y una población urbana que busca apropiarse de un nuevo espacio reproduciendo las comodidades de la ciudad.

No obstante, el aumento de la renta del suelo ocasionado por la expansión de las infraestructuras, de los servicios y las amenidades rurales en las subregiones del Altiplano y de Embalses está generando un acelerado proceso de segregación, situación exacerbada por las desigualdades sociales y el conflicto armado y la que puede, eventualmente, generar diferencias y conflictos entre nativos, residentes permanentes y nuevos pobladores.

Todo lo anterior permite indicar que las poblaciones rurales del Este de Antioquia tienen unas relaciones y conciencias socio-espaciales diferenciadas y con esto a la vez aparecen procesos de segregación socio espacial -al interior de las zonas rurales por localidad- a raíz de las desigualdades sociales y como efecto de la producción de la renta del suelo rural.

Ahora bien, es necesario subrayar que, para el caso de esta región, el desplazamiento, el abandono-despojo y el retorno de campesinos es un determinante ya que, así como hay familias que se desplazaron, también se encuentran otras familias que no se desplazaron de sus lugares de residencia, pero que, durante más de una década, vieron sus vidas "consternadas".

Si bien el conflicto armado, y con este la migración forzada, ha determinado en gran medida los saldos poblacionales de las localidades de las subregiones de Páramos y Bosques en las últimas dos décadas, se encuentra que la migración por amenidades y la movilidad residencial de las zonas urbanas hacia las zonas rurales en la subregión del Altiplano y de Embalses ha sido una constante.

Ambos procesos, la migración forzada y la migración por amenidades, están determinando la actual geografía humana de la región del Este de Antioquia. Y en esta región parece persistir, según los datos de distribución de la propiedad rural entre subregiones, una desigualdad creciente de oportunidades, tanto para las poblaciones de las zonas rurales como a nivel subregional: el Altiplano y Embalses son la subregiones con mayores ventajas en infraestructura vial e hidroeléctrica, mientras que Bosques y Páramos las más afectadas por el conflicto armado. 
La propiedad y la renta rural se expresan en un desigual acceso a servicios básicos en las zonas rurales, a una segregación espacial y diferenciación social en aumento y, en sí, a una brecha social cada vez más grande entre pobladores rurales del Altiplano y de Embalses (rural cercano) con los pobladores rurales de Bosques y Páramos (rural profundo).

\section{Referencias bibliográficas}

AGUDELO, L. Ruralidad Metropolitana. Entre la tradición rural y el "brillo" urbano. Una interpretación. Bulletin d L'Institut Français d'Études Andines, 2012, Vol. 3, № 41, p. 555-571.

ARANGO, G. Salvemos el espacio rururbano colombiano. Cuadernos de vivienda y urbanismo, 2008, Vol. 2, No 1, p. 394-409.

ÁVILA, H. Tendencias recientes en los estudios de Geografía rural. Desarrollos teóricos y líneas de investigación en países de América Latina. Investigaciones Geográficas, Boletín del Instituto de Geografía, 2015, № 88, p. 75-90.

BAIGORRI, A. ¿Ruralia de nuevo? (Desarrollo local en el marco de la urbanización global). En: GARCÍA DOCAMPO, M. (editor). Perspectivas teóricas en desarrollo rural. La Coruña: Netbiblo, 2007, p. 193-231.

BARROS, C. La ciudad en el campo: Nuevas ruralidades y lugares rururbanos. En: Nogué, J. \& Romero, J. (editores). Las otras geografías. Valencia: Tirant Lo Blanch, 2006, p. 325- 338.

BONILLA, R. Urbanización rural y economía agrícola de sobrevivencia en la Delegación Milpa Alta. Argumentos, Revista Argumentos, 2008, Vol. 27, № 74, p. 195-215.

CADAVID, G. M. Ruralidad en contextos metropolitanos, un desafío en procesos de planeación, ordenamiento territorial y gestión. Revista Soluciones de Postgrado EIA, 2009, № 4, p. 243-266.

CASTAÑEDA, Y. Familias campesinas y rurales en el contexto de la nueva ruralidad. Estudio de caso en la vereda del Hato del municipio de la Calera. Bogotá: Tesis Doctoral, Universidad Nacional de Colombia, 2012.

CASTAÑO, Y. Rurales Metropolitanos: La Emergencia de un Nuevo Grupo Social. Medellín: Tesis de Maestría, Universidad Nacional de Colombia, 2014.

CLEMENTI, L. Del nostálgico recuerdo al creciente entusiasmo por lo rural: indicios de la revalorización y el retorno a los espacios rurales. GeoGraphos. Revista Digital para Estudiantes de Geografía y Ciencias Sociales, 2012, Vol. 3, Nº. 30, p. 25.

CRAVIOTTI, C. Tensiones entre una ruralidad productiva y otra residencial: el caso del partido de Exaltación de la Cruz, Buenos Aires, Argentina. Revista Economía, Sociedad y Territorio, 2014, Vol. $6, N^{\circ} 23$, p. $745-772$. 
CRUZ, B. N. Procesos de gentrificación en lugares rururbanos: presupuestos conceptuales para su estudio en Colombia. En: Territorialidades Na América Latina. Goiânia: Centro Editoral y Grafico de la Universidad Federal de Goiás, 2009, p. 134-148.

DEPARTAMENTO NACIONAL DE ESTADÍSTICA. Censo Nacional de Población y Vivienda, 2005. Disponible en Internet: http://www.dane.gov.co.

ENTRENA DURÁN, F. Cambios en la construcción social de lo rural. De la autarquía a la globalización. Madrid: Tecnos, 1998.

ENTRENA DURÁN, F. La ruralidad en España: de la mitificación conservadora al neorruralismo. Cuadernos de desarrollo rural, 2012, Vol. 9, N69, p. 39-65.

FAJARDO, D. Las Zonas de Reserva Campesina: ¿Estrategia de desarrollo regional y contra el desplazamiento. 2000. Disponible en Internet:

http://www.prensarural.org/spip/spip.php?article4066

FERNÁNDEZ, O. Entre la evasión y la nostalgia. Estrategias de la neoruralidad desde la economía social. Gazeta de Antropología, 2013, Vol. 29, № 2, p. 9.

FERNÁNDEZ, D.; MORENO, M. y RUIZ, D. Tras las huellas de la ancestralidad Muisca en tres municipios de Cundinamarca como fuente de saber pedagógico: ejercicio investigativo. Bogotá: Tesis de Grado, Universidad Pedagógica Nacional, 2013.

GARCÍA, C. \& ARAMBURO, C. Geografías de la guerra, el poder y la resistencia: Oriente y Urabá antioqueños 1990-2008. Medellín: INER, 2011.

GARCÍA, E. \& GÁLVEZ, A. La gestión pública ambiental rural, un tema abierto al debate. Revista Gestión y Ambiente, 2011, Vol. 14, № 2, p. 73-84.

GIARRACA, N. (compilador). ¿Una nueva ruralidad en América Latina? Buenos Aires: CLACSO, 2001.

GIARRACA, N. América Latina, nuevas ruralidades, viejas y nuevas acciones colectivas. En: Giarracca, N. y Levy, B. (compiladoras). Ruralidades latinoamericanas. Identidades y luchas sociales. Buenos Aires: CLACSO, Consejo Latinoamericano de Ciencias Sociales, 2004, p. 13-43.

GOBERNACIÓN DE ANTIOQUIA, Departamento Administrativo de Planeación. Perfil Subregional Oriente Antioqueño. 2009. Disponible en Internet: http://www.dane.gov.co

GONZÁLEZ, J.L. Condominios y estabilidad ecológica en los andes centrales colombianos. Revista Luna Azul, 2009, Vol. 29, № 29, p. 54-67.

GONZÁLEZ, R.; OTERO, A.; NAKAYAMA, L. y MARIONI, S. Las movilidades del turismo y las migraciones de amenidad: problemáticas y contradicciones en el desarrollo de centros turísticos de montaña. Revista de Geografía Norte Grande, 2009, № 44, p. 75-92. 
GUIMOND, L. \& SIMARD, M. Les néo--ruraux et les ruraux de longue date sont--ils si différents au Québec? Analyse de leur mobilité, sens des lieux et engagement. Journal of Regional Science, 1998, Vol. 34, Nº 4, p. 151-163.

GUIMOND, L. \& SIMARD, M. Gentrification and neo-rural populations in the Québec countryside: Representations of various actors. Journal of Rural Studies, 2010, Vol. 26, N 4, p. 449-464.

HINES, J. Rural gentrification as permanent tourism: the creation of the New'West Archipelago as postindustrial cultural space. Environment and Planning D: Society and Space, 2010, $\mathrm{N}^{\circ} 28, \mathrm{p}$. 509-525.

HINES, J. The Post-Industrial Regime of Production/Consumption and the Rural Gentrification of the New West Archipelago. Antipode, 2012, Vol. 44, № 1, p. 74-97.

INSTITUTO DE ESTUDIOS REGIONALES, Universidad de Antioquia. Oriente. Desarrollo regional: una tarea común. Universidad- Región. Medellín: Universidad de Antioquia, 2000.

KAYSER, B. Ils ont choisi la campagne. La Tour d'Aigues: Editions de l'Aube, Collection Poche Essai, 1996.

LLAMBÍ, I. y PÉREZ, E. Nuevas ruralidades y viejos campesinismos. Agenda para una nueva sociología rural en América Latina. Cuadernos de desarrollo rural, 2007, №59, p. 37-61.

MARTÍNEZ, C. Las migraciones internas en Colombia. Análisis territorial y demográfico según los censos de 1973 y 1993. Barcelona: Tesis Doctoral, Universidad Autónoma de Barcelona, 2002.

MÉNDEZ, M. El neorruralismo como práctica configurante de dinámicas sociales alternativas: un estudio de caso. Revista Luna Azul, 2012, №34, p. 113-130.

MÉNDEZ, M. Una tipología de los nuevos habitantes del campo: aportes para el estudio del fenómeno neorrural a partir del caso de Manizales, Colombia. Revista de Economía de Sociología Rural, $2013, N^{\circ} 51$, p. 31-48.

MERLOTTO, A.; PICCOLO, M.C. \& BÉRTOLA, G. R. Crecimiento urbano y cambios del uso/ cobertura del suelo en las ciudades de Necochea y Quequén, Buenos Aires, Argentina. Revista de Geografía Norte Grande, 2012, N 53, p. 159-176.

MORALES, E. Los nuevos pobladores en el medio rural de Castilla y León. Valladolid: Tesis Doctoral, Universidad de Valladolid, 2016.

NATES, B. \& RAYMOND, S. Buscando la naturaleza. Migración y dinámicas rurales contemporáneas. Barcelona: Anthropos Editorial, 2007.

NATES, B. Proceso de gentrificación en lugares rururbanos: presupuestos conceptuales para su estudio en Colombia. Antropología Sociológica, 2008, Vol. 10, p. 253-269. 
NATES, B. Pensar el mundo, practicar el entorno. Etnografías y reflexiones desde una antropología de las territorialidades. Revista de Dialectología y Tradiciones Populares, 2009, Vol. LXIV, N 1, p. 277-296.

ORTIZ, J. \& HERNÁNDEZ, Y. Analysis of Local Perceptions and Responses to the Process of Rurbanization in the Municipal Subdivision of Chuntame, Municipality of Cajicá, Cundinamarca, on the Basis of Local Knowledge. Cuadernos de Geografía: Revista Colombiana de Geografía, 2015, Vol. 24, $N^{\circ} 1$, p. 87-99.

PARSONS, J. Exploración y descubrimiento en geografía. Geografía en Español, 2005, № 2, p. 1-17. Disponible en Internet: http://www.geografiaenespanol.net/Parsons-2005.pdf

PÉREZ, E. El mundo rural latinoamericano y la nueva ruralidad. Nómadas (Col), 2004, N²0, p. 180-193.

PÉREZ, E.; FARAH, M. y CARTON DE GRAMMONT, H. La nueva ruralidad en América Latina. Avances teóricos y evidencias empíricas. Bogotá: Pontificia Universidad Javeriana, 2008.

PERRIER-CORNET, P. Repenser les campagnes. París: Éditions de l'Aube, 2002.

PRADILLA, E. Campo y ciudad en el capitalismo actual. Ciudades, 2002, № 54, p. 219-250.

RAMÍREZ, B. La vieja agricultura y la nueva ruralidad: enfoques y categorías desde el urbanismo y la sociología rural. Sociológica, 2003, № 51, p. 49-71.

RED NACIONAL DE INFORMACIÓN. Registro Único de Victimas (RUV). 2015.

RUIZ, N. El Desplazamiento forzado en el interior de Colombia: caracterización sociodemográfica y pautas de distribución territorial 2000-2004. Barcelona: Tesis Doctoral, Universidad Autónoma de Barcelona, 2008.

RUIZ, N. y DELGADO, J. Territorio y nuevas ruralidades: un recorrido teórico sobre las transformaciones de la relación campo-ciudad. EURE, 2008, Vol. 34, № 102, p. 77-95.

SALAZAR, A.; DÍAZ MERY, O.; OSSES MC-INTYRE, P. y FOSTER BONNETTE, W. Relevancia de la accesibilidad a los centros urbanos, con respecto a la pobreza y ocupación de la población rural y periurbana de la región del Bío-Bío, Chile. Cuadernos de Geografía: Revista Colombiana de Geografía, 2013, Vol. 23, № 2, p. 221-238.

UNIDAD PARA LA ATENCIÓN Y REPARACIÓN INTEGRAL DE VÍCTIMAS. Registro Nacional De Información. Registro Único de Víctimas (RUV). 2016. Disponible en Internet: http://rni.unidadvictimas. gov.co/?q=node/107

URIBE, M. Desplazamiento forzado en Antioquia. Aproximaciones teóricas y metodológicas al desplazamiento de población en Colombia. Bogotá: Conferencia Episcopal de Colombia, Secretariado Nacional de Pastoral Social, 2001. 